\title{
Achievable Performance Improvements Provided by Cooperative Diversity
}

\author{
Stephan Bohacek* \\ University of Delaware \\ Newark, DE 19716 \\ bohacek@udel.edu
}

\begin{abstract}
This paper examines the achievable performance gains when a particular class of cooperative relaying known as best-select is employed. In essence, best-select seeks to improve performance by exploiting diversity. We closely examine a particular topology when the number of nodes is finite as well when the node density goes to infinity. Determining the performance is complicated by the correlation of the channel gains between nearby channels (i.e., when the receivers are nearby and the transmitters are nearby). This challenge is overcome by modeling the channels as spatially continuous diffusion processes and as a random field and then using a Poisson clumping heuristic. In the example network and metric studied, it is found that in the limit, node cooperation can provide an increase in the end-toend performance by $35 \mathrm{~dB}$ (i.e., a factor of $\sim 3000$ ) in a 5-hop network. This result motivates further work in the design of multihop cooperative protocols.
\end{abstract}

\section{INTRODUCTION}

One of the most important features of mobile wireless networks is the variability of channels. In traditional wireless networking, great pains are taken to mitigate the impact of the variability of channels. While all layers must cope with the effects of time-varying channels, there has been extensive effort at the network layer. For example, there are a number of techniques that seek to find precomputed backup paths (e.g. [1]). Thus, when a primary path fails, a new route search is not required to find a new path. However, time-varying channels does not only imply that links may break, it also implies that some links are better than others. Indeed, in the context of communication theory, channel diversity means that there may be some channels between the same transmitter and receiver (but with different antennas) that have better performance than other channels. This diversity is closely related to the stochastic nature of channels. Specifically, if channel gains can be modeled as a set of independent random variables, then the larger the set, the higher the probability that one channel will provide good performance. While communication theory provides a clear picture of diversity between a single transmitter and single receiver (each with

\footnotetext{
* This work was prepared through collaborative participation in the Collaborative Technology Alliance for Communications and Networks sponsored by the U.S. Army Research Laboratory under Cooperative Agreement DAAD1901-2-0011. The U.S. Government is authorized to reproduce and distribute reprints for Government purposes notwithstanding any copyright notation thereon.
}

one or more antennas) (e.g., Chapter 11 of [2]), diversity in the setting of multihop wireless networks is only recently receiving attention. This paper explores the benefits that may be obtained when diversity is fully exploited within multihop wireless networks. This paper focuses only on the performance potential. Questions about how such diversity can be exploited and the cost in terms of overhead and congestion is left for future work and is partially investigated in [3] and [4].

In this paper, it will be shown that diversity may provide tremendous benefits. For example, in the environment explored here, we find that diversity can lead to end-to-end performance gains of up to a factor of 3000 across a 5 hop network (the definition of the metric is provided in the next section). At first glance, an improvement of a factor of several thousand seems unreasonably large. However, one must consider that dynamic range of a physical layer such as 802.11 exceeds $50 \mathrm{~dB}$. That is, a good channel may be $50 \mathrm{~dB}$ better than a bad channel, i.e., a good channel may be 100000 times better than a bad channel. The critical challenge for communication theorist or practitioner is to achieve communication even when the channel is bad. However, at the network layer, there is the option of seeking to use other better channels. If these alternative channels are sufficiently good, then one might well expect an improvement of several orders of magnitude. Indeed, in this paper, such improvements are found.

This paper proceeds as follows. In the next section, the problem is stated and basic terminology defined. In Section III, the performance is found where the correlation between channels is neglected. In this section, the node occupancies (which will be defined later) are may be dependent. Section III-A presents a short discussion of a computationally efficient approximation that assumes that the node occupancies are independent. Based on this approximation, Section III-B examines several different topologies. Interestingly, it is observed that the improvement offered by diversity is not greatly impacted by the topologies. In Section IV, the performance is determined when the channels are correlated. Accommodating correlated channels allows one to consider the case when the number of nodes goes to infinity. This section is based on modeling the channels as an Ornstein-Uhlenbeck process and then employing the Poisson Clumping Heuristic [5]. 
It should be noted that this work is closely related to the active research in cooperative communication, including [6], [7], [8], [9], [10], [11], [12], [13]. However, here the focus is on multihop networks.

\section{Problem Definition And Terminology}

The goal of diversity exploiting routing schemes is to utilize alternative routes that provide better performance than an arbitrarily selected one. To make this problem more specific, consider the topology shown in Figure 1. In this paper we will explore the benefit of allowing to use alternative paths besides the nominal route, which consists of using relays along the center (i.e., the shortest geographic path). Alternatively, we explore the possibility of using the path that is best in terms of a particular route metric. Here the route metric of interest is the maximum channel loss ${ }^{1}$ along the route. That is, each link of a path has a particular channel loss, and the route metric is the maximum of these link channel losses. To put it another way, the route metric is the channel loss of the worst link along the route. This paper examines the performance gain that is achieved by using a route that is optimal in terms of this route metric as compared to the nominal route.

Since the received signal strength is proportional to the transmitted power divided by the channel loss, the channel loss is closely related to the SNR. Hence, minimizing the maximum channel loss is the same as maximizing the minimum SNR experience by each receiver along the route. There are several motivations for trying to minimizing the maximum channel loss. For example, in most physical layer schemes, the probability of a transmission error across a link falls to zero quickly as the SNR increases beyond a threshold. Thus, the probability of successfully delivering a packet to a destination along a route is dominated by the probability of successfully transmitting a packet over the worst link along the route. Similarly, if the transmission power at each link is adjusted so that a target SNR is met (e.g., to meet a target link transmission error probability), then the energy to deliver a packet from the source to destination is dominated by the energy required to transmit across the worst link along the path. Also, if the bit-rate across each link is maximized under the constraint that the transmission error probability is less than a specified threshold, then the throughput along the route is given by the bit-rate across the slowest link, which, again, is the worst link along the route in terms of channel gain. See [13], for further examination of metrics.

In order to compute the average performance, we compute the cumulative probability function of the route metric along the best path. We denote this probability ${ }^{2}$ with $U$ i.e.,

$U(r):=P$ (there exists a path with min channel loss $<r$ ).

\footnotetext{
${ }^{1}$ The channel loss is the recipocal of the channel gain. Hence, a good channel has a small channel loss.

${ }^{2}$ It is more convenient to use channel loss rather than channel gain.
}

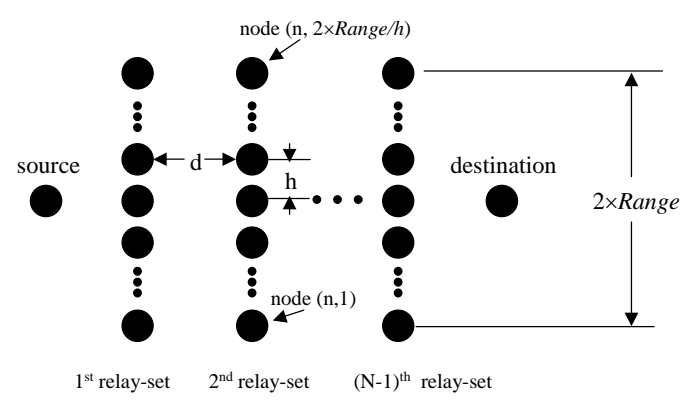

Fig. 1. The objective is to find the best path from source to destination. The nominal path is made up of the nodes along the center. In this paper, we explore the impact of being allowed to use any combination of nodes. However, each hop must be between two nodes in adjacent relay-sets and the route must always proceed toward the destination. The distance between the relay-sets is $d$, and the space between adjacent nodes is $h$. Each relay-set spans a vertical distance $2 \times$ Range.

We employ the terminology of percolation. Fixing $r$, we say that a link is open if the channel loss is less than $r$, and closed otherwise. A node is said to be occupied if there is a sequence of open links from the source to the node. Thus, $U(r)$ is the probability that the destination is occupied.

This paper focuses on the topology shown in Figure 1. The figure shows the source and destination as well as several sets of nodes that are made up of nodes placed in a vertical line. As indicated in the figure, these sets are called relaysets. The relay-sets are numbered with the first relay-set being closest to the source. It is assumed that each transmission carries the packet from a node in the $n$-th relay-set to a node in the $(n+1)$-th relay-set, but the exact node within each relay-set that relays the packet can be adjusted. As is also indicated in Figure 1, the nodes are labeled with node $(n, i)$ being the $i$-th node in the $n$-th relay-set. We assume that the number of nodes in each relay-set is fixed, and is sometimes denoted as $M$. Sometimes it is convenient to think of the source as a node in the zeroth relay-set, i.e., $(0,\lfloor M / 2\rfloor)$, where $\lfloor M / 2\rfloor$ is the largest integer that is less than or equal to $M / 2$. Similarly, the destination is $(N,\lfloor M / 2\rfloor)$. Note that sometimes it is convenient to denote the number of nodes in each relay-set as $\lfloor 2 \times$ Range $/ h\rfloor$ rather than $M$.

We assume that the channel loss is composed of a deterministic part that depends on the distance between the transmitter and receiver and a random part that has a lognormal distribution. Specifically, let $(n, i)$ be the $i$ th node in the $n$th relay-set as indicated in Figure 1 . The probability that the link from $(n, i)$ to $(n+1, j)$ is open is denoted by $q_{\text {Thresh }}(|i-j|)$ where

$$
\begin{aligned}
& q_{\text {Thresh }}(|i-j|):= \\
& P\left(\text { Thresh }>X+2.7 \times 10 \log _{10}\left(\sqrt{d^{2}+h^{2}(i-j)^{2}}\right)\right)
\end{aligned}
$$

where $X$ is Gaussian with mean 0 and standard deviation $\sigma$, and Thresh such that if the channel loss is less than Thresh, 
then transmission can be decoded. Here the attenuation factor is set to 2.7 [14]. Other possible values range from 1.6 to 4. Note that we focus on shadow-fading as modeled with by a log-normal random variable [14]. We do not consider fastfading or Rayleigh fading. One reason for this is that in wide bandwidth communication, the bandwidth is wide enough that the impact of fast-fading is limited [14]. Nonetheless, future work will consider the impact of fast-fading (e.g., [3]). (1) does not indicate the correlation of the channel loss between nearby channels (i.e., channel where the sources and destinations are nearby). This will be considered in Section IV.

\section{IndePendent Channels And Dependent Node OCCUPANCIES}

One way to determine the performance is to represent the occupied nodes in a relay-set as a Markov chain where the state of the Markov chain is the vector of which nodes are occupied or not occupied. Then $a \in\{0,1\}^{M}$ is an element in the state-space of the Markov chain where there are $M$ nodes in each relay-set. In particular $a_{i}=1$ implies that the $i$ th node is occupied and $a_{i}=0$ implies that the $i$ th node is not occupied. The state can be represented more succinctly as an integer between 0 and $2^{M}-1$, i.e., $A=\sum_{i=1}^{M} a_{i} 2^{i}$. Then the probability transition matrix for this Markov chain is

$$
\begin{aligned}
& Q_{\text {Thresh }}(A, B):= \\
& P(\text { moving from state } A \text { to state } B) \\
= & \prod_{\left\{i: b_{i}=1\right\}}\left(1-\prod_{\left\{j: a_{j}=1\right\}}\left(1-q_{\text {Thresh }}(|i-j|)\right)\right) \\
& \times \prod_{\left\{i: b_{i}=0\right\}}\left(\prod_{\left\{j: a_{j}=1\right\}}\left(1-q_{\text {Thresh }}(|i-j|)\right)\right)
\end{aligned}
$$

To understand (2), note that the probability of node $i$ not being occupied is the probability that each link from every occupied node in the previous relay-set is closed, which is $\prod_{\left\{j: a_{j}=1\right\}}\left(1-q_{\text {Thresh }}(|i-j|)\right)$ where $\left\{j: a_{j}=1\right\}$ is the set of nodes that are occupied. Furthermore, the set of nodes that are occupied in state $B$ is $\left\{i: b_{i}=1\right\}$. Thus, the probability of being in state $B$ is the probability that each node $i$ is occupied if $b_{i}=1$ and each node with $b_{i}=0$ is not occupied.

From (2), it is straightforward to compute the probability distribution of the occupied nodes within the $n$-th relay-set. Specifically, we can think of the source as the $\lfloor M / 2\rfloor$-th node within the 0 -th relay-set where $\lfloor M / 2\rfloor$ is $M / 2$ rounded down to the nearest integer. Thus, we set the probability distribution of the occupied nodes within the 0-th relay-set to be $V$ with $V_{A}=1$ if $A=2^{\lfloor M / 2\rfloor}$. The probability distribution of of the set of occupied/unoccupied nodes $n$ hops from the source is $V Q_{\text {Thresh }}^{n}$, where $Q_{\text {Thresh }}$ is a $2^{M} \times 2^{M}$ matrix.

The destination can be thought of as the $\lfloor M / 2\rfloor$-th node

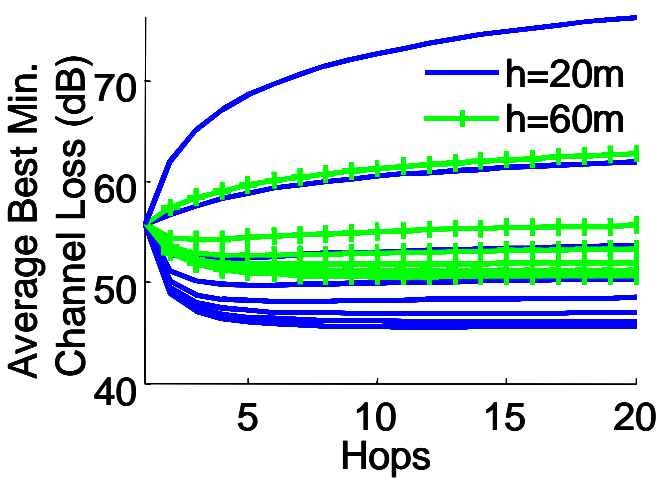

Fig. 2. Average maximum channel loss along the best path. The solid curves are for the case when $h=20 \mathrm{~m}$ and the marked curves are for $h=60 \mathrm{~m}$. The upper most curve is when there is only one node in each relay-set, i.e., when diversity is not exploited. This curve is the same regardless of the value of $h$. As the size of the relay-sets grows, the maximum channel loss along the best path decreases. This figure shows the performance for relay-set size of $1,2,4,6, \cdots, 14$. The best performance is indicated by the lowest curve and corresponds to the case where $M=14$.

in the $N$-th relay-set. Given that the probability distribution in the $N$ th relay-set is $U$, the probability that the $\lfloor M / 2\rfloor$ is occupied is found by summing the elements of $U$ over all the states that have the $\lfloor M / 2\rfloor$ th node occupied. Specifically, set $W$ to be a row vector with $W_{A}=1$ if $a_{\lfloor M / 2\rfloor}=1$ where $A=\sum_{i=1}^{M} a_{i} 2^{i}$. Then the probability that the destination is occupied is

$$
\Gamma(\text { Thresh })=V \times Q_{\text {Thresh }}^{N} \times W .
$$

Finally, from (3) the probability distribution of the maximum channel loss along the best route from the source to destination can be determined. Figure 2 shows the expected value of the maximum channel loss along the best path as a function of the number of hops from source to destination where the number of nodes within the relay-set is $1,2,4, \ldots 14$ and distance between neighboring nodes within a relay-set is $h=20 \mathrm{~m}$ and $h=60 \mathrm{~m}$. In this case, the standard deviation of the shadow fading was $11 \mathrm{~dB}$ (i.e., $\sigma=11 \mathrm{~dB}$ ) and the distance between relay-sets was $100 \mathrm{~m}$ (i.e., $d=100 \mathrm{~m}$ ). Note that the $M=1$ case is the case when there is only one path from source to destination. Thus, the $M=1$ case corresponds to when diversity is not exploited and hence represents the benchmark performance.

In Figure 2, the largest relay-set size is 14. In this case, there are $2^{14}$ elements in the state-space and the state transition matrix has $2^{28}$ elements ${ }^{3}$. With the processors available, this was the largest topology whose performance could be computed in a realistic amount of time.

\footnotetext{
${ }^{3}$ It is possible to compute the probability distributions without computing the entire state transition matrix.
} 
A. Independent Channel and Independent Node Occupancy An Approximation

A dramatic improvement in computation can be obtained by assuming that the occupancy of a node is independent of whether other nodes within the same relay-set are also occupied. It will be shown that this independence assumption provides an upper bound on performance. Furthermore, it will be observed that for large relay-sets, this independence assumption does not have a significant impact on the accuracy.

First we compute the probability of occupancy under the independence assumption. Let the probability that the $j$-th node in the $n$-th relay-set is occupied be $P_{n, \text { Thresh }}(j)$. Then probability that node $i$ in the $(n+1)$-th relay-set is occupied is

$$
\begin{aligned}
& P_{n+1, \text { Thresh }}(i) \\
= & 1-\prod_{j=1}^{M}\left(\left(1-P_{n, \text { Thresh }}(j)\right)\right. \\
& \left.+\left(1-q_{\text {Thresh }}(|i-j|)\right) P_{n, \text { Thresh }}(j)\right) \\
= & 1-\prod_{j=1}^{M}\left(1-q_{\text {Thresh }}(|i-j|) P_{n, \text { Thresh }}(j)\right) .
\end{aligned}
$$

As above, the fact that the source is occupied is expressed by $P_{0, \text { Thresh }}(i)=1$ for $i=\lfloor M / 2\rfloor$ and 0 otherwise and note that the probability of there exists a path from source to destination such that the maximum channel loss is less than Thresh is $P_{N, T h r e s h}(\lfloor M / 2\rfloor)$, which can be easily computed from 4.

This approximation yields performance relationships that are quite similar to those shown in Figure 2, and hence is not shown. Instead, consider Figure 3, which shows the difference between the expected performance found using (4) and (2). Observed that for large networks the error is less than $1 \mathrm{~dB}$ and converges as the length of the path grows. For smaller networks, the error is larger, but for small relay-set sizes, an approximation is not needed since the actual performance can be easily computed. It can also be noticed that the error is always positive implying that the approximation is always larger than the actual value. This is always the case as explained next.

Proposition 1: The assumption that the event that a node is occupied is independent of the event that other nodes within the same relay-set are occupied is leads to an upper bound on performance.

Proof: Let $U_{i}$ be the event that node $(n, i)$ is occupied and node $i$ has and open link to node $(n+1, k)$. Then the

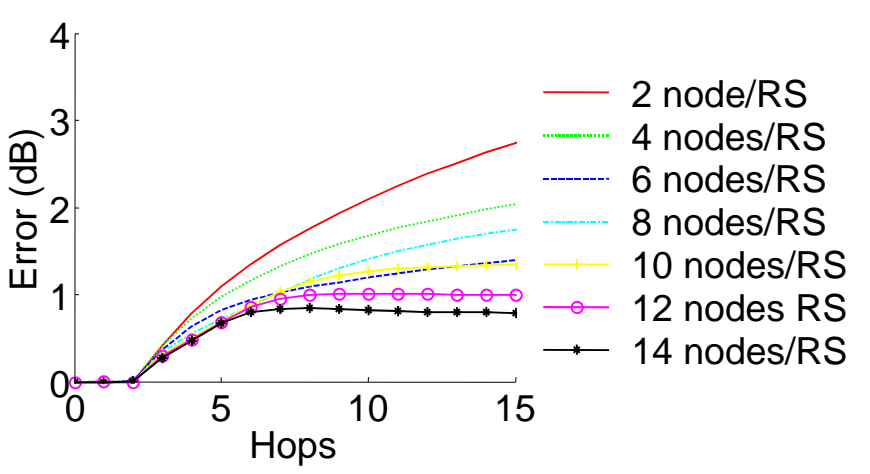

Fig. 3. The difference between the exact value of the optimal value of the route metric and an approximation where the approximation assumes that the occupancy of a node in a relay-set is independent of the occupancy of other nodes within the same relay-set. This figure shows this error for networks with 2 - 14 nodes per relay-set and for networks with 2 to 20 hops. Note that an error of $0 \mathrm{~dB}$ means that there is no error and an error of $3 \mathrm{~dB}$ means that the approximation suggests a performance that is $3 \mathrm{~dB}$ better than the actual performance. The results here are for $d=100 \mathrm{~m}$ and $h=20 \mathrm{~m}$.

probability that node $(n+1, k)$ is occupied is $P\left(\bigcup_{i} U_{i}\right)$ and

$$
P\left(\bigcup_{i} U_{i}\right)=\sum_{i} P\left(U_{i}\right)-\sum_{i \neq j} P\left(U_{i} \cap U_{j}\right) .
$$

Under the assumption that the events $U_{i}$ are independent

$$
\tilde{P}\left(\bigcup_{i} U_{i}\right)=\sum_{i} P\left(U_{i}\right)-\sum_{i \neq j} P\left(U_{i}\right) P\left(U_{j}\right),
$$

where $\tilde{P}$ denotes the probability under the independence assumption. However, since the events $U_{i}$ are increasing,

$$
P\left(U_{i} \cap U_{j}\right) \geq P\left(U_{i}\right) P\left(U_{j}\right) \text {. }
$$

Thus $\tilde{P}\left(\bigcup_{i} U_{i}\right) \geq P\left(\bigcup_{i} U_{i}\right)$. For further discussion on increasing events see Chapter 2 of [15].

\section{B. Performance for Some Different Topologies}

Using (4), it is computationally tractable to consider a wide variety of topologies. For example, suppose that $n$th relay-set consists of a strip of nodes centered along the line $(n \times d, y)$ for $-\infty<y<\infty$. Or more generally, for $-d / 2<x<d / 2$ and $-\infty<y<\infty$ the $n$th relay-set includes a node at location $(n \times d, 0)+(x, y)$ with probability $\rho_{n}(x, y)$ (setting $\rho_{n}(x, y)$ to 0 or 1 provides a fixed deterministic topology). Then (4) can be generalized to

$$
\begin{aligned}
& P_{n+1, \text { Thresh }}(x, y)=\rho_{n}(u, v) \times(1- \\
& \left.\prod_{\substack{-d<2<u<d / 2 \\
-\infty<v<\infty}}\left(1-q_{G}(x-u, v-y) P_{n, \text { Thresh }}(u, v)\right)\right) .
\end{aligned}
$$




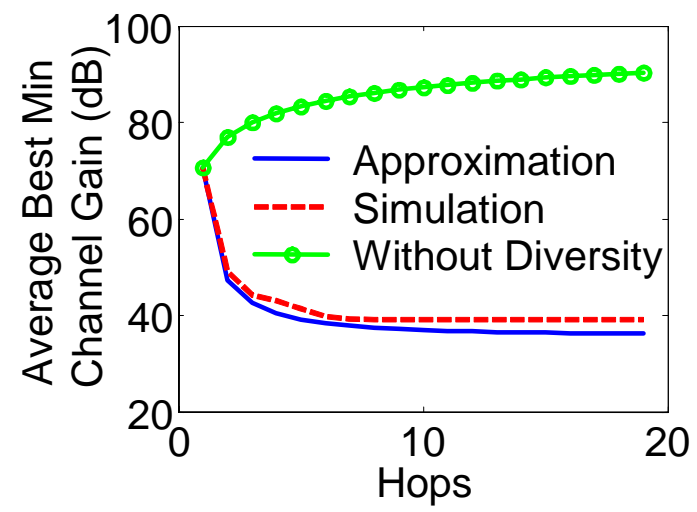

Fig. 4. Performance when nodes within the $n$th relay-set are distributed between $(n \times d, 0)+(10 i+10 j)$ for $0<i, j<40, d=400$. The upper curve is the performance when diversity is not used, i.e., $M=1$. The lowest curve is found with (6), while the middle curve is from Monte Carlo simulation. Note that the simulation and approximation closely match.

where

$$
\begin{aligned}
& q_{G}(x-u, v-y):=P(\text { Thresh }> \\
& \left.X+2.7 \cdot 10 \log _{10}\left(\sqrt{(d+x-u)^{2}+(v-y)^{2}}\right)\right),
\end{aligned}
$$

with $X \sim N(0,11)$.

With (6) we explore the performance for some topologies other than the one shown in Figure 1. First we consider the performance when the $n$th relay-set is composed of a set of nodes located at $(n \times d, 0)+(i h, j h)$ for $-d / h<i, j<d / h$, i.e., the relay-set is the lattice of nodes centered around $(n \times d, 0)$ with spacing between node $h$. Figure 4 shows the performance for $d=400$ and $h=10$. For reference two other curves are also shown. The upper most curve corresponds to the performance when diversity is not used (i.e., $M=1$ ), while the two lower curves show different estimates of the performance when the relay-sets are as just described. The lowest curve was found using (6) while the second to the lowest was found from simulations. This plot confirms the appropriateness of the approximation (4) and (6). This curves also shows that the improvement in performance exceeds 40 $\mathrm{dB}$ at 5 hops.

Next we compare performance several topologies. As oppose to most other examples in this paper, the relay-sets in these examples are $400 \mathrm{~m}$. apart. Referring to Figure 5, (a) horizontal 1 and (b) horizontal 2 have relay-sets configured into horizontal lines (as oppose the vertical lines as in Figure 1). (a) horizontal 1 has the nodes uniformly spaced, where as (b) horizontal 2 has the nodes more clustered around the center relay-set. Specifically, in (a) horizontal 1 the $i$ th node in the $n$th relay-set is located at $(n 400,0)+\left(\frac{400}{M} i, 0\right)$ where there are $M$ nodes in the relay-set, and in (b) horizontal 2 the $i$ th node of the $n$th relay-set is located at $(n 400,0)+(10(M / 2+i), 0)$. The vertical topologies are similar, the $i$ th node in the $n$th relay-set in (c) vertical 1 is located at $(n d, 0)+\left(0, \frac{400}{M} i\right)$, and the $i$ th node the relay-set in (d) vertical 2 is located at $(n d, 0)+(0,10(M / 2+i))$. The last topology, (e) rectangle, has the nodes in a rectangle clustered around the center of the relay-set. In this case the nodes in the relay-set are indexed by two integers, $i$ and $j$, with the node $i, j$ located at $(n d, 0)+$ $(10 i-5 \sqrt{M}, 10 j-5 \sqrt{M})$ with $i, j=0,1, \cdots, \sqrt{M}$. Since the computation does not account for correlation between channels, it is important that the nodes be spaced far enough apart so that correlation is insignificant. We assume that if the nodes are farther than $10 \mathrm{~m}$. apart, then correlation is small (see the next section for more discussion of the correlation between channels). This condition is maintained if $M \leq 40$ in the first and third topology, and if $M \leq 40^{2}$ in the fifth topology. In the second topology if $M>40$, then the relaysets overlap and correlations must be considered. There are no restrictions on $M$ in the fourth topology.

Figure 5 shows the expected improvement provided by diversity in these topologies. More specifically, it shows the average value of the maximum channel gain along the best path (in $\mathrm{dB}$ ) minus the average maximum channel gain along the path that only used nodes located at $(n 400,0)$. As indicated, three path lengths are shown. While the topologies are quite varied, it is surprising to observe that the performance as a function of the number of nodes in each relay-set are quite similar for all topologies. One important implication is that while the topology shown in Figure 1 is only a specific topology, other topologies show similar performance, hence Figure 1 is able to provide insight into the performance of these other topologies.

\section{Correlated Channels And IndePendent Node OCCUPANCIES}

The left-hand plot in Figure 2 shows that the performance improves when either the size of the relay-sets increases or when the spacing between nodes within the relay-set decreases. Both of these trends are reasonable. As the size of the relay-set increases, there are more paths available and hence the probability that a good path is available increases. As the spacing between nodes within a relay-set decreases, the quality of the paths from any single node to all the nodes in the next relay-set is improves. Specifically, recall that the distance between nodes $(n, i)$ and $(n+1, j)$ is $\sqrt{d^{2}+h^{2}(i-j)^{2}}$ which decreases with $h$, the spacing between nodes within the same relay-set (see Figure 1). Based on the trends shown in Figure 3, the question naturally arises as to what is the maximum performance that can be achieved by increasing the number of nodes in the relay-sets and by decreasing the spacing between nodes within a relay-set. In this section this question is answered. However, a major difficulty is that as $h$ decreases, the channels become correlated. For example, as $h$ decreases, the nodes $(n, i)$ and $(n, i+1)$ are closer together. Hence, the channel between $(n, i)$ and $(n+1, j)$ and the channel between $(n, i+1)$ and $(n+1, j)$ past through the 

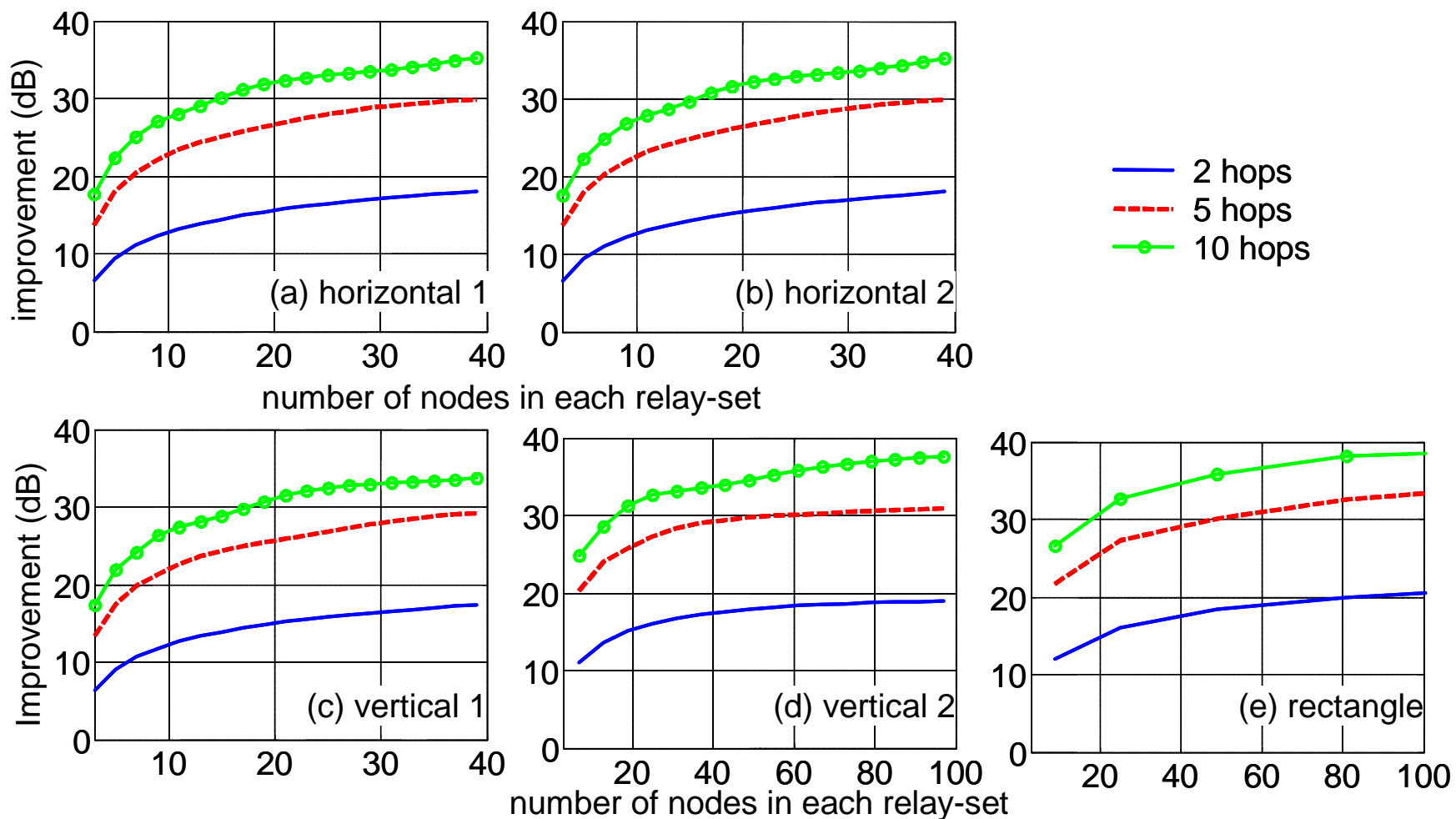

Fig. 5. Performance offered by diversity for several different topologies. See the text for a discussion of the topologies examined.

same environment, are subject to the same impairments, and therefore, have the similar loss.

The approach here models the relay-sets as a continuum of nodes and models the channel as a diffusion process or a random field. Then the Poisson clumping heuristic is used to approximate the performance. Before examing the performance of multihop networks, we first consider the 2-hop network and then consider the channels between two relaysets. In the first case, the channel is modeled as a diffusion process and in the second case it is modeled as a random field.

\section{A. Two Hop Case}

Correlated channels subject to shadow-fading have been reasonably well studied [16], [17], [18], and this investigation applies models from this previous work. Specifically, we employ a diffusion-based model of the channel loss similar to what is developed in [16]. Suppose that the relay-set spans from location $(d,-$ Range $/ 2)$ to $(d$, Range $/ 2)$. Let the shadow-fading part of the channel loss (in $\mathrm{dB}$ ) from the source located at $(0,0)$ to the node located at $(d, y)$ be given by $L_{y}^{1}$ where - Range $\leq y \leq$ Range. Define $L_{y}^{2}$ similarly but for the shadow-fading from the node at location $(d, y)$ to the destination at location $(2 d, 0)$. Thus, there is two hops between the source and destination. The stochastic part of the channels are modeled as Ornstein-Uhlenbeck process, specifically,

$$
\begin{aligned}
& d L_{y}^{1}=-\alpha L_{y}^{1} d y+\sigma \sqrt{2 \alpha} d B_{y}^{1}, \\
& d L_{y}^{2}=-\alpha L_{y}^{2} d y+\sigma \sqrt{2 \alpha} d B_{y}^{2},
\end{aligned}
$$

where $B_{y}^{i}$ are Brownian motion processes with $B^{1}$ and $B^{2}$ independent. It has been observed through measurements that $\alpha \approx \frac{1}{10} \mathrm{~m}^{-1}$ [18]. Furthermore, while $\sigma$ may range from 4 to $12 \mathrm{~dB}$, we use $\sigma=11 \mathrm{~dB}$. Note that (7) implies that $L_{y} \sim N(0, \sigma)$ and

$$
E\left(L_{y} L_{x}\right)=\sigma^{2} \exp (-\alpha(|y-x|)) .
$$

Therefore, in the limit, as the node density goes to infinity, the probability that there exists a path from source to destination such that each link has a channel loss that is lower than Thresh is given by

$$
\begin{aligned}
& U_{\infty}(\text { Thresh }, \text { Range }):= \\
& \quad P(\exists \text { a } y \text { such that }- \text { Range }<y<\text { Range, } \\
& L_{y}^{1}+2.7 \times 10 \log _{10}\left(\sqrt{d^{2}+y^{2}}\right)<\text { Thresh }, \\
& \text { and } \left.L_{y}^{2}+2.7 \times 10 \log _{10}\left(\sqrt{d^{2}+y^{2}}\right)<\text { Thresh }\right)
\end{aligned}
$$

While such probabilities are difficult to exactly compute, a first order estimate can be easily found as long as Thresh is small enough. In particular, if Thresh is small, then the probability of the event occurring rare and can be approximated with the Poisson clumping heuristic (See [5] for details). Specifically, a 2-hop path such that both haps have a channel loss less 


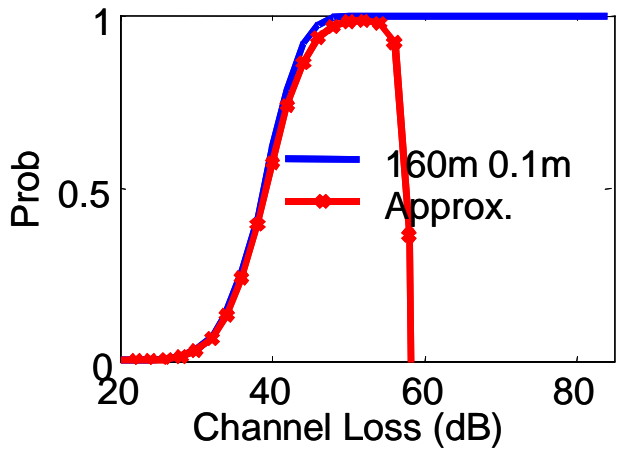

Fig. 6. Discrete channel simulated and continuous approximation of the cumulative distribution of the maximum channel loss along the best path over a 2-hop network. The discrete channel simulations used nodes spaced $0.1 \mathrm{~m}$ apart. In both estimates, the relay-sets were $160 \mathrm{~m}$. long. The relay-sets were $100 \mathrm{~m}$ apart.

than Thresh is a nonhomogeneous Poisson process with rate $\lambda(y)$. Thus, the probability of suitable path passing through any relay located between -Range and Range is approximately $\hat{U}_{\infty}$ (Thresh, Range), where

$$
\hat{U}_{\infty}(\text { Thresh, Range })=1-\exp \left(\int_{- \text {Range }}^{\text {Range }} \lambda(y) d y\right) .
$$

The rate $\lambda$ is given by

$$
\lambda(y)=-\alpha u(y) \times 2 \phi_{0, \sigma}(u(y))\left(1-\Phi_{0, \sigma}(u(y))\right),
$$

where $-\alpha u(y)$ is the drift in (7), $\phi_{0, \sigma}(u)$ is the probability density of a Gaussian random variable with 0 mean and standard deviation $\sigma, \Phi_{0, \sigma}(u)$ is the cumulative distribution of a Gaussian random variable with mean 0 and standard deviation $\sigma$, and

$$
u(y)=2.7 \times 10 \log _{10}\left(\sqrt{d^{2}+y^{2}}\right)-\text { Thresh } .
$$

Thus, $2 \phi_{0, \sigma}(u(y))\left(1-\Phi_{0, \sigma}(u(y))\right) \delta y$ is approximately the probability of the channel loss being in a small region around where two-hop route metric exceeds Thresh (i.e., the channel loss of both channels from the source to the node at position $y$ and from node at $y$ to the destination are both below Thresh).

Figure 6 show values of $\hat{U}_{\infty}$ (Thresh, Range) and estimates values of $U_{\infty}$ (Thresh, Range) for Range $=160 \mathrm{~m}$. The estimate to $U_{\infty}$ models the continuum of channels with discrete set of channels. We call this a discrete channel estimation as compared to $\hat{U}_{\infty}$, which is an approximation of the continuum of channels.

The discrete channel estimation can be found with Monte Carlo simulations. To this end, we define $V^{1}$ to be a realization of the channel losses from the source to the relay-set and $V^{2}$ to be a realization the channel losses to the destination from the relay-set. Thus, $V_{i}^{1}$ is the channel loss from the source to the node at $(d,-$ Range $+h i)$ and $V_{j}^{2}$ is channel loss from the node at $(d,-$ Range $+h i)$ to the destination. Note that
$V^{1}=H^{T} W^{1}+C$ where, in this case $H^{T} H$ is the Cholesky factorization of $\sum$ with $\sum_{i, j}=\sigma^{2} \exp (-\alpha h|i-j|)$ and $C_{i}=2.7 \times 10 \log _{10}\left(\sqrt{d^{2}+(- \text { Range }+h i)^{2}}\right)$ and $W^{1}$ is vector of uncorrelated Gaussian random variables with zero mean and variance of one. $V^{2}$ is generate in the same way. Finally, the realization contains a suitable path if there exists an $0 \leq i \leq 2 \times$ Range $/ h$ such that $V_{i}^{1}<$ Thresh and $V_{i}^{2}<$ Thresh. Figure 6 shows the cumulative distribution of the maximum channel loss along the best path for $h=0.1$. Furthermore, for this fine spacing of nodes, the approximation $\hat{U}_{\infty}$ of the $U_{\infty}$ is close to the discrete channel estimate if Thresh is small enough. The large error for large Thresh is expected since the approximation is only for rare events.

\section{B. Channels Between Two Relay-Sets}

Before using the Poisson clumping heuristic to estimate the performance across a multihop network, we examine the performance between two relay-sets. In this case, the correlation between channels is slightly more complicated. Specifically, consider the channels between nodes located at $(n d, u)$ and $((n+1) d, x)$ and between $(n d, v)$ and $((n+1) d, y)$. If $x \approx y$ and/or $u \approx v$, then these channel will be correlated. Let $L(u, x)$ be the stochastic part of the channel between $(n d, u)$ and $((n+1) d, x)$. Then [19]

$$
\begin{aligned}
& E(L(u, x) L(v, y)) \\
& =\sigma^{2} \exp (-\alpha(|u-v|+|x-y|))
\end{aligned}
$$

Note that (10) becomes (8) if $u=v$.

It is clear that $L$ is a random field, or more specifically, $L$ is a product Ornstein-Uhlenbeck process [5]. That is, $L$ is a two parameter process (as oppose the $L$ in the previous section which was a single parameter process) with $E(L(u, x) L(v, y)) \approx \sigma^{2}(1-\alpha|u-v|-\alpha|x-y|)$ for $\alpha|u-v|+\alpha|x-y|$ small.

Let $U_{\infty}^{R R}$ (Thresh, Range) be the probability that there exists a channel between the two relay-sets that has channel loss less than Thresh, or

$$
\begin{aligned}
& U_{\infty}^{R R}(\text { Thresh, Range }):=P(\exists x \text { and } y \text { such that } \\
& \quad-\text { Range }<x, y<\text { Range and } L(x, y)<\text { Thresh }) .
\end{aligned}
$$

The Poisson clumping heuristic can also be applied to product Ornstein-Uhlenbeck processes. We denote the approximation of $U_{\infty}^{R R}$ as $\hat{U}_{\infty}^{R R}$ and, as above, our approximation models $U_{\infty}^{R R}$ with a nonhomogeneous Poisson distribution, i.e.,

$$
\begin{aligned}
& \hat{U}_{\infty}^{R R}(\text { Thresh, Range }) \\
= & 1-\exp \left(\int_{- \text {Range }}^{\text {Range }} \int_{- \text {Range }}^{\text {Range }} \lambda(x, y) d x d y\right) .
\end{aligned}
$$


In this case,

$$
\lambda(x, y)=\alpha^{2}(u(x, y))^{3} \phi_{0,1}(u(x, y)),
$$

where

$u(x, y)=\frac{1}{\sigma}\left(2.7 \times 10 \log _{10}\left(\sqrt{d^{2}+(x-y)^{2}}\right)-\right.$ Thresh $)$.

Figure 7 shows $\hat{U}_{\infty}^{R R}$ (Thresh, Range) along with a discrete channel estimate of $U_{\infty}^{R R}$. The discrete channel estimate is generate in a similar fashion as above, but is significantly more computational complex. Specifically, for $0 \leq i \leq$ 2 Range/ $h$ and $0 \leq j \leq 2$ Range/2, let $V_{i \times 2 \text { Range } / h+j}$ be the channel loss from the node located at $(n d,-$ Range $+h i)$ to the node located at $((n+1) d,-$ Range $+h j)$. Then define the correlation matrix

$\Sigma_{i \times 2 \text { Range } / h+j, k \times 2 \text { Range } / h+l}:=\sigma^{2} \exp (-\alpha(|i-k|+|j-l|))$

Then

$$
V_{i \times 2 \text { Range } / h+j}=H^{\prime} W+C
$$

where $H^{T} H$ is the Cholesky factorization of $\Sigma$, $C_{i \times 2 \text { Range } / h+j}=2.7 \times 10 \log _{10}\left(\sqrt{d^{2}+(h i-h j)^{2}}\right)$, and $W$ vector of uncorrelated Gaussian random variables with zero mean and variance of one. Thus, if there is an $i, j$ such that $V_{i \times 2 \text { Range } / h+j}<$ Thresh, then there is a channel between the two relay-sets that has channel loss less than Thresh.

In Figure 7, Range $=10 \mathrm{~m} ., h=0.3 \mathrm{~m}$. , and $d=$ $100 \mathrm{~m}$. In this case there are 60 nodes in each relay-set, and hence there are $60^{2}$ channels. Thus, $\Sigma$ is a $60^{2} \times 60^{2}$ matrix, which is nearly the largest matrix for which Cholesky factorization can be easily performed (e.g., with Matlab). On the other hand, Range $=10$, is a rather small relay-set and $h=0.3$ is not particularly fine (Recall that in Figure 6 $h=0.1$ and Range $=160$.). Nonetheless, Figure 7 shows reasonable agreement between the two estimates. For example, the medians differs only by about $3 \mathrm{~dB}$. Again, since the approximation is only valid for rare events, there is large error for Thresh large.

\section{Performance Over a Multihop Network}

Combining the above techniques, it is possible to approximate the performance over a multihop network. Specifically, the technique developed in Section IV-A is used for the first and last hop, and the technique developed in Section IV-B is used for intermediate hops. Following similar notation as above,

$$
\begin{aligned}
& U_{\infty}^{M H}(\text { Thresh, Range }):=P(\text { there exists a path } \\
& \text { with maximum channel loss }<\text { Thresh }) .
\end{aligned}
$$

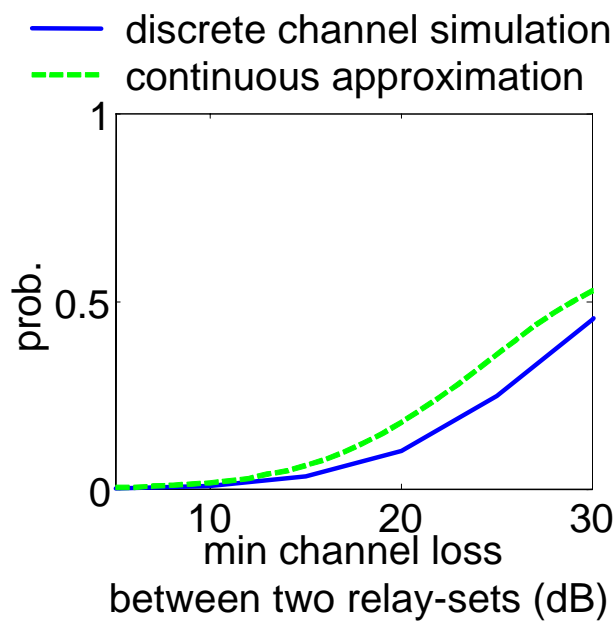

Fig. 7. Cumulative distribution of the maximum channel gain between two relay-sets. The relay-sets were $100 \mathrm{~m}$. apart and $20 \mathrm{~m}$. long. The discrete simulation approximated the relay-set with nodes spaced $0.3 \mathrm{~m}$. apart. The continuous approximation used the Poisson clumping heuristic.

We approximate $U_{\infty}^{M H}$ with $\hat{U}_{\infty}^{M H}$, where

$$
\begin{aligned}
& \hat{U}_{\infty}^{M H}(\text { Thresh, Range })=1- \\
& \exp \left(\int_{- \text {Range }}^{\text {Range }} \cdots \int_{- \text {Range }}^{\text {Range }} \lambda\left(x, y_{2}, \cdots y_{N-1}, z\right)\right. \\
& \left.d x d y_{2}, \cdots d y_{N-2} d z\right) .
\end{aligned}
$$

where

$$
\begin{aligned}
& \lambda\left(x, y_{2}, \cdots y_{N-1}, z\right) \\
& =\alpha u(x) \phi_{0, \sigma}(u(x)) \Phi_{0,1}^{c}\left(u\left(x, y_{2}\right)\right) \cdots \\
& \times \Phi_{0,1}^{c}\left(u\left(y_{N-2}, y_{N-1}\right)\right) \Phi_{0,1}^{c}\left(u\left(y_{N-1}, z\right)\right) \Phi_{0, \sigma}^{c}(u(z)) \\
& +\alpha^{2}\left(u\left(x, y_{2}\right)\right)^{3} \phi_{0,1}\left(u\left(x, y_{2}\right)\right) \Phi_{0, \sigma}^{c}(u(x)) \Phi_{0,1}^{c}\left(u\left(y_{2}, y_{3}\right)\right) \cdots \\
& \times \Phi_{0,1}^{c}\left(u\left(y_{N-2}, y_{N-1}\right)\right) \Phi_{0,1}^{c}\left(u\left(y_{N-1}, z\right)\right) \Phi_{0, \sigma}^{c}(u(z)) \\
& +\cdots \\
& +\alpha^{2}\left(u\left(y_{N-2}, z\right)\right)^{3} \phi_{0,1}\left(u\left(y_{N-2}, z\right)\right) \Phi_{0, \sigma}^{c}(u(x)) \\
& \times \Phi_{0,1}^{c}\left(u\left(x, y_{2}\right)\right) \cdots \Phi_{0,1}^{c}\left(u\left(y_{N-3}, y_{N-2}\right)\right) \Phi_{0, \sigma}^{c}(u(z)) \\
& +\alpha u(z) \phi_{0, \sigma}(u(z)) \Phi_{0, \sigma}^{c}(u(x)) \Phi_{0,1}^{c}\left(u\left(x, y_{2}\right)\right) \cdots \\
& \times \Phi_{0,1}^{c}\left(u\left(y_{N-2}, y_{N-1}\right)\right) \Phi_{0,1}^{c}\left(u\left(y_{N-1}, z\right)\right)
\end{aligned}
$$

with $\Phi_{\mu, \sigma}^{c}(x)=1-\Phi_{\mu, \sigma}(x), u(x)$ is given by (9), $u(x, y)$ is given by (11), and $N$ is the number of hops between the source and destination.

Following techniques discussed in the previous sections, it is possible the estimate $U_{\infty}^{M H}$ with a discrete channel estimate. Of course, this technique suffers from the drawback that it can only be performed on topologies with only a moderate number of nodes in each relay-set (e.g., less than 60 nodes per relay-set). Figure 8 shows the cumulative distribution of the maximum channel loss along the best path over a 3-hop network. Here Range $=10 \mathrm{~m}$., and $d=100 \mathrm{~m}$. Two estimates are shown, $\hat{U}_{\infty}^{M H}($ Thresh, 20$)$ and the discrete channel estimate of $U_{\infty}($ Thresh, 20$)$, where the discrete channel estimate 


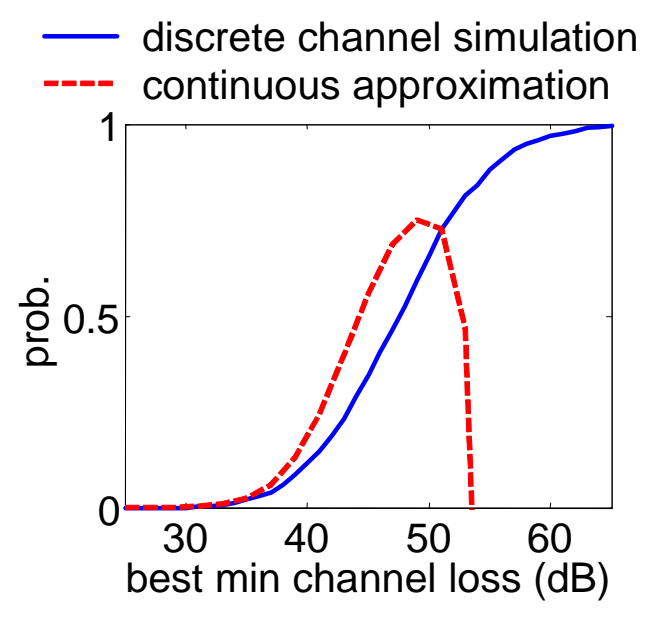

Fig. 8. Cumulative distribution of the maximum channel loss over the best path over a 3-hop network. The continuous approximation used the Poisson clumping heuristic.

had $h=0.3 \mathrm{~m}$. As above, the two estimate are in reasonable agreement when Thresh is small. For example, the medians are within approximately $3 \mathrm{~dB}$ of each other.

The performance for larger values of Range and more hops can be approximated via $\hat{U}_{\infty}^{M H}$. Figure 9 shows the median value of the maximum channel loss along the best path for several values of Range and for networks with up to 5 hops with $d=100 \mathrm{~m}$. Note that due to the difficulty of computing high dimensional integrals, the longest paths examined had only 5 hops.

Figure 9 shows that as the Range increases, the performance improvement due to adding more nodes decreases. This is reasonable since the added nodes are quite far away from the geometric minimum path. On the other hand, when Range $=80$, there are still some improvements possible by including nodes that are quite far from the geometric minimum path. While not shown here, improvements were observed for the 2-hop network when Range was allowed to grow to 100 $\mathrm{m}$. Considering that the relay-sets are $100 \mathrm{~m}$ apart, this shows that improvements can be achieved by considering paths with a geometric length ${ }^{4}$ that is $40 \%$ longer then the path with shortest geometric length.

For reference purposes, the maximum channel gain along the path with shortest geometric length is shown. We see that for the topology examined here, diversity allows nearly $35 \mathrm{~dB}$ of improvement over the path shortest geometric length. Thus, in this case, diversity improves the performance by a factor of approximately 3000 .

\footnotetext{
${ }^{4}$ The geometric length of a path is the sum the distances between adjacent
}

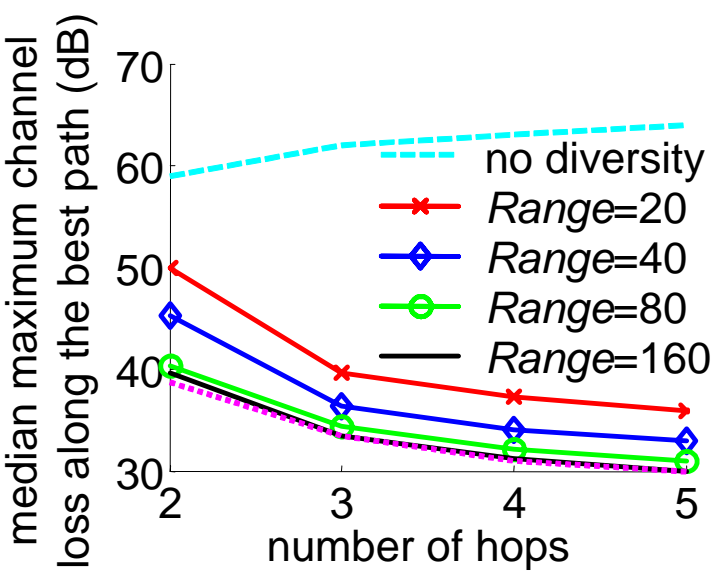

Fig. 9. Achievable performance provided by diversity for the topology shown in Figure 1.

\section{COnCLusions}

The performance impact of diversity over multihop networks was examined. Several techniques for determining the performance were developed. The first method assumes that the node spacing was large enough so that channel correlation could be ignored. However, this method is computationally difficult and can accommodate at most 14 nodes per relayset. An approximation of this method was developed that was shown to provide an upper bound on performance. However, the bound appears to be quite good yielding an error in the expect performance of $1 \mathrm{~dB}$ for networks with large relay-sets. While approach can be applied to a variety of topologies, it is required that the nodes are spaced far enough apart that channel correlations can be ignored. In order to examine the performance for high node densities, the correlation must be accounted for. This was accomplished by modeling the relaysets as a continuous set of nodes, and hence the channels were modeled as a continuum of channels. In this case the channels are modeled as diffusion processes (specifically, a OrnsteinUhlenbeck processes) or random fields (specifically, a product Ornstein-Uhlenbeck processes). The Poisson clumping heuristic was used to approximate the performance in this case. For the topology examined, it was found that diversity can improve performance (in terms of the route metric considered) by about $35 \mathrm{~dB}$.

One future direction is to examine the performance in more general topologies, but with very dense nodes. In this case, the channel from location $(w, x)$ to $(y, z)$ is written $L(w, x, y, z)$, hence the channels are a 4parameter random field. And $E(L(s, t, u, v) L(w, x, y, z))=$ $\sigma^{2} \exp \left(-\alpha\left(\sqrt{(s-w)^{2}+(t-x)^{2}}+\sqrt{(u-y)^{2}+(v-z)^{2}}\right)\right)$. The Poisson clumping heuristic must be extended to examine the performance of such channels. 


\section{DISCLAIMER}

The views and conclusions contained in this document are those of the authors and should not be interpreted as representing the official policies, either expressed or implied, of the Army Research Laboratory or the U. S. Government.

\section{REFERENCES}

[1] M. K. Marina and S. R. Das, "Ad hoc on-demand multipath distance vector routing," tech. rep., SUNY - Stony Brook, 2003.

[2] J. R. Barry, E. A. Lee, and D. G. Messerschmitt, Digital Communication. Boston: Kluwer Academic Publishers, 2004.

[3] S. Bohacek, R. Blum, L. Cimini, L. Greenstein, and A. Haimovich, "The impact of the timeliness of information on the performance of multihop best-select," in Military Communications Conference (Milcom), 2005.

[4] J. Kim and S. Bohacek, "Enhancing routing performance by exploiting diversity," in Submitted, 2005.

[5] D. Aldous, Probability Approximations Via the Poisson Clumping Heuristic. New York: Springer Verlag, 1989.

[6] A. Sendonaris, E. Erkip, and B. Aazhang, "User cooperation diversity - Part II: Implementation aspects and performance analysis," IEEE Transactions on Communication, vol. 51, pp. 1939-1948, 2003.

[7] J. L. Neman, D. Tse, and G. Wornell, "Cooperative diversity in wireless networks: Efficient protocols and outage behavior," IEEE Transactions on Information Theory, To appear.

[8] J. N. Laneman, G. W. Wornell, and D. N. C. Tse, "An efficient protocol for realizing cooperaivte diversity in wireless networks," in Proceedings of the IEEE International Symposium on Information Theory, (Washington, D.C.), p. 294, 2001.

[9] J. Luo, R. S. Blum, L. J. Greenstein, L. J. Cimini, and A. M. Haimovich, "New approaches for cooperative use of multiple antennas in ad hoc wireless networks," in Proceedings of the IEEE Vehicular Technology Conference (VTC '04-Fall), 2004.

[10] B. Zhao and M. C. Valenti, "Practical relay networks: A generalization of hybrid-ARQ," IEEE Journal on Selected Areas in Communications, vol. 23, pp. 7-18, 2005.

[11] J. Boyer, D. D. Falconer, and H. Yanikomeroglu, "Multihop diversity in wireless relaying channels," IEEE Transactions on Communication, vol. 3, no. 6, pp. 1963-1968, 2004.

[12] S. Biswas and R. Morris, "ExOR: Opportunistic multi-hop routing for wireless networks," in Sigcomm05, 2005.

[13] J. Kim and S. Bohacek, "Selection metrics for multihop cooperative relaying," in Proceeding of the Fourth Annual Mediterranean Ad Hoc Networking Workshop (MedHoc), 2005.

[14] T. Rappaport, Wireless Communication. Pearson Education, 2002.

[15] G. Grimmett, Percolation. berlin: Springer, 1999.

[16] C. D. Charalambous and N. Menemenlis, "A state-space approach in modeling multipath fading channels via stochastic differential equations," in IEEE International Conference on Communication (ICC), pp. 11-14, 2001.

[17] R. Vijayan and J. M. Holtzman, "Foundations for level crossing analysis of handoff algorithms," in ICC, 1993.

[18] M. Gudmundson, "Correlation model for shadow fading in mobile radio systems,” Electron. Lett., vol. 27, pp. 2145-2146, 1991.

[19] F. Graziosi and F. Santucci, "A general correlation model for shadow fading in mobile radio systems," IEEE Communication Letters, vol. 3, pp. 102-104, 2002. 